\title{
Computação desplugada como instrumento avaliativo no Ensino Superior: um relato de experiência
}

\author{
Sara das Mercês Silva ${ }^{1}$, Abner Cardoso $^{2}$, Maria Meninéa Duarte ${ }^{3}$, \\ Fabíola Pantoja O. Araujo ${ }^{1}$, Josivaldo Araújo ${ }^{1}$, Marcos César da R. Seruffo ${ }^{3}$ \\ ${ }^{1}$ Instituto de Ciências Exatas e Naturais (ICEN) \\ Universidade Federal do Para (UFPA) \\ Rua Augusto Corrêa, 01 - 66075-110 - Belém - PA - Brasil \\ ${ }^{2}$ Instituto Tecgraf \\ Pontifícia Universidade Católica do Rio de Janeiro \\ Rua Marquês de São Vicente, 225 - 2245190 - Rio de Janeiro - RJ - Brasil \\ ${ }^{3}$ Programa de Pós-Graduação em Estudos Antrópicos na Amazônia (PPGEAA) \\ Universidade Federal do Pará (UFPA) \\ Av. Maximino Porpino da Silva, 1000 - 68740-080 - Castanhal- PA - Brasil \\ maru.merces18@gmail.com, acardoso@tecgraf.puc-rio.br, menineamaria@gmail.com, \\ fpoliveira@ufpa.br, josivaldo@ufpa.br, seruffo@ufpa.br
}

\begin{abstract}
This study presents a quantitative-qualitative research, which describes the experiences and results of the application of activities, in the unplugged perspective, which served as an evaluative method to Database II discipline of the Bachelor of Computer Science course at the Federal University of Pará. The results show, although the students did not have prior knowledge or the practice of Unplugged Computing, they had a performance 29\% higher in relation to the grade when compared to those who took an objective test. In addition, the active evaluation methodology was shown to be positive for $60 \%$ of students who showed satisfaction in experiencing the new, to the detriment of traditional methods.
\end{abstract}

Resumo. Este estudo apresenta uma pesquisa quantitativa, onde descreve as experiências e resultados da aplicação de atividades, na perspectiva desplugada, que serviram como método avaliativo para a disciplina de Banco de Dados II do curso de Bacharelado em Ciência da Computação da Universidade Federal do Pará. Os resultados indicam que, apesar dos estudantes não terem conhecimento prévio e nem a prática da Computação Desplugada, eles tiveram um rendimento $29 \%$ maior em relação a nota, quando comparado com àqueles que realizaram prova objetiva. Além disso, a metodologia ativa de avaliação, mostrou-se positiva para $60 \%$ dos alunos que externaram satisfação ao vivenciarem ao novo, em detrimento aos métodos tradicionais.

\section{Introdução}

As mudanças advindas dos avanços das tecnologias digitais de informação e comunicação (TDICs) e a rapidez na troca de novos conhecimentos que a sociedade como um todo vem presenciando, estão atingindo as mais diferentes áreas de trabalho e pesquisa 
[Assis 2015]. Sendo assim, o modo de ensinar e aprender precisa ser aprimorado e adequado às necessidades latentes de discentes e docentes em todas as esferas de ensino, inclusive na graduação.

Isso não é diferente quando reporta-se aos estudantes de Computação, que estudam diversas disciplinas as quais exigem abstração para representar problemas reais, prática do raciocínio lógico, aprendizando de algoritmos, dentre outras habilidades. Nesse contexto, as metodologias ativas (MA) surgem forma de ensinar e aprender, inclusive dentro dos cursos de ensino superior em Computação. Em [Hartwig et al. 2019], afirmase que nas MA, o aluno é o protagonista do aprendizado e o professor incentiva-o a aprender e a construir seu conhecimento por meio da informação disponível. As MA abrangem diferentes maneiras de ensinar e aprender e considera-se exemplos dela, o uso da Computação Desplugada (CD) e atividades em grupo [Lázaro et al. 2018].

A Computação Desplugada caracteriza-se por ser um método para ensinar Computação, ou o Pensamento Computacional, sem o uso do computador, objetivando desenvolver atividades interativas para os estudantes, tornando a sala de aula e os assuntos mais atrativos, consequentemente, melhorando o processo de ensino aprendizagem [Curzon et al. 2014]. Nesse sentido, aplicar CD como um método de ensinoaprendizagem, ou mesmo avaliativo, dentro da Computação, pode ser uma alternativa para estimular a abstração, o pensamento e raciocínio lógico dos estudantes, ajudandoos assim na capacidade de resolução de problemas [Bell et al. 2011]. No trabalho de [Reis et al. 2018], afirma-se que na literatura existem três principais tipos de pesquisa sobre o uso da $\mathrm{CD}$ no processo de ensino e aprendizagem de Computação: a adaptação das atividades desplugadas (AD) do livro Computer Science Unplugged [Bell et al. 2011] com foco em grupos específicos, construção de novas atividades desplugadas para diversos conteúdos de Computação e a junção da técnica de $\mathrm{CD}$ a outras metodologias ou abordagens. Neste trabalho utilizou-se a junção de CD com outra abordagem, no caso o trabalho em grupo, assim como foram desenvolvidas ADs para conteúdos da disciplina de Banco de Dados II (BD II).

Desta forma, este artigo tem por objetivo relatar a experiência de aplicação da Computação Desplugada no ensino superior, como método avaliativo na disciplina de BD II, em uma turma de bacharelado em Ciência da Computação da Universidade Federal do Pará - campus Belém, assim como analisar se trabalhos em grupos e a interação entre os alunos contribuem para um melhor desempenho, como relata [Lázaro et al. 2018] em sua pesquisa sobre Metodologias Ativas. Optou-se pela dinâmica em grupo, visto que uma característica da cultura do nativo digital, que deve ser aplicada no ensino, é a sua predileção por realizar atividades em cooperação, desenvolvendo de forma colaborativa o conteúdo ensinado [Chris and Shao 2011].

O trabalho está estruturado da seguinte forma: na Seção 2 são apresentados os trabalhos relacionados; na Seção 3 é descrita a metodologia usada neste trabalho e o detalhamento das atividades aplicadas em sala de aula; na Seção 4 são apresentados os resultados obtidos e, por fim, na Seção 5, são apresentadas as considerações finais.

\section{Trabalhos Relacionados}

Há na literatura alguns relatos da utilização da Computação Desplugada em alguns conteúdos no ensino superior. Os cursos com perspectivas maiores de introdução 
dessas atividades são os de Exatas, especificamente os da área da Computação [Souza et al. 2019]. Pesquisas e estudos aplicando a CD em disciplinas específicas da Computação vêm sendo desenvolvidos no Brasil. Em [Gonçalves et al. 2013] foram realizadas atividades dentro da disciplina Fundamentos e Metodologias do Ensino de Informática na Educação I, no curso de Licenciatura em Computação. Além de utilizar a ferramenta Scratch, foi criado o objeto de aprendizagem desplugado denominado EvOU, sendo o objetivo dessa aula ensinar os conectivos lógicos E, OU e NÃO, pertencentes ao conteúdo de lógica computacional, e a montagem de suas expressões. Os autores afirmam que essa metodologia possibilita um contato maior com a realidade do ensino da informática, onde o docente adquire maiores experiências práticas e amplia os métodos de ensinar a programação.

A pesquisa de [Silva et al. 2018] relata o uso da Computação Desplugada em conjunto com MA para promover o processo de ensino-aprendizagem na disciplina de Redes de Computadores. Os autores ensinaram conteúdos sobre firewall e filtros de pacotes, realizaram uma dinâmica prática utilizando cartas confeccionadas pelos discentes. Através da avaliação diagnóstica, o professor analisou o desempenho dos alunos por toda a atividade e os alunos escreveram um texto relacionando à atividade prática e conteúdo técnico. Em [Alejandro Melgaço 2019] apresenta-se um relato de experiência em que a CD também foi aplicada no ensino de Redes de Computadores. Neste trabalho, foram construídos objetos de aprendizagem desplugados para serem utilizados nos seguintes assuntos: funções básicas de dispositivos usados em Redes de Computadores e Modelo OSI. Um dos objetos desplugados construídos foi o Tabulosi, um jogo de tabuleiro elaborado. A análise aplicada evidenciou que os objetos desplugados promoveram um ensino mais atrativo para os discentes. O trabalho de [Moreira and Monteiro 2018] expõe uma experiência realizada utilizando a gamificação e a CD para ensinar Estrutura de Dados no curso de Licenciatura em Computação. As atividades foram realizadas através de "missões" nas quais os desafios eram os alunos criarem atividades desplugadas para o ensino de recursividade, pilha, fila, lista encadeada e árvore de decisão.

Quanto ao uso da Computação Desplugada no ensino da disciplina Banco de Dados, existem pesquisas nacionais aplicadas ao ensino básico e superior [Berardi et al. 2019, Martinhago et al. 2014]. O trabalho de [Berardi et al. 2019] apresenta a experiência de uma oficina de Banco de Dados onde foi adaptada a atividade Human Branching Databases para ensinar Banco de Dados às meninas através da construção de árvores binárias. Segundo os autores e as avaliações realizadas por meio de questionários, a experiência mostrou resultados positivos no uso desse tipo de prática para divulgar e motivar meninas na área de Computação. O trabalho de [Martinhago et al. 2014] desenvolveu um projeto para a disciplina Banco de Dados no curso Sistemas de Informação, onde foram aplicadas atividades baseadas na Computação Desplugada em uma turma denominada grupo desplugado (GD) e outra grupo controle (GC). Foram selecionados conceitos de banco de dados. As avaliações foram feitas por meio de questionários. Os resultados mostraram que a metodologia desplugada não trouxe uma aprendizagem significativa com relação aos conceitos, porém foi uma proposta positiva quanto à satisfação dos alunos. Em relação a usar CD como método avaliativo, em [Berto et al. 2019] são desenvolvidas atividades para reforçar conceitos aplicados nas atividades plugadas e para avaliação formativa do aprendizado, visando acompanhar a evolução e focando na eficácia do aprendizado. 
Os trabalhos apresentados nessa seção mostram o uso da CD no ensino de disciplinas específicas da Computação, no ensino básico e superior brasileiro. Algumas metodologias assemelham-se àquelas aplicadas ao presente estudo, pois conforme em alguns trabalhos aqui relatados, nesta pesquisa os alunos foram divididos em grupos [Gonçalves et al. 2013, Martinhago et al. 2014] para desenvolverem as atividades desplugadas, porém estas atividades serviram de avaliação para os discentes, permitindo uma comparação quantitativa com os métodos tradicionais de avaliação (provas individuais).

\section{Metodologia}

O artigo apresenta uma pesquisa aplicada e quantitativa, visto que trata-se de uma experiência prática, objetivando avaliar aprendizados através de atividades desplugadas em uma disciplina na graduação, onde levar-se-á em conta, na avaliação e resultados, informações quantitativas [Prodanov and Freitas 2013]. As atividades desenvolvidas serviram como método avaliativo na disciplina de BD II, em uma turma de 36 alunos, do sexto semestre do curso de Bacharelado em Ciência da Computação. A cronologia dos eventos consistiu em: aulas teóricas (expositivas) sobre conteúdos da referida disciplina e assuntos abordados no projeto, exercícios (teóricos e práticos), duas atividades desplugadas e duas provas objetivas.

As aulas da disciplina aconteceram duas vezes por semana (1:40h por aula) durante dois meses. As aulas teóricas foram ministradas pela professora, os exercícios e as atividades desplugadas contaram com o auxílio de duas alunas de mestrado, em estágio de docência. Após as aulas teóricas, os alunos resolveram listas de exercícios práticos. Antes das atividades desplugadas, as alunas de mestrado ministraram uma aula para a turma sobre o que são Metodologias Ativas e Computação Desplugada, pois esse conteúdo não é contemplado em BD II. A disciplina era composta por três avaliações e este projeto foi desenvolvido durante a segunda avaliação, a qual teve a atividade desplugada, durando em média duas horas e meia a três horas, e uma prova teórica-objetiva, realizada durante uma hora e quarenta minutos.

Devido à complexidade dos assuntos, a segunda avaliação foi dividida em duas partes (partes 1 e 2) e a turma de discentes também foi dividida em dois grandes grupos (G1 e G2), sendo cada um deles composto por 16 alunos. As avaliações aconteceram em dois dias de aula diferentes. No primeiro dia, foram realizadas a avaliação teórica e a atividade desplugada sobre o conteúdo referente à parte 1 . O G1, que realizou prova objetiva, ficou em uma sala de aula com uma das alunas de mestrado, enquanto o G2 participou da atividade desplugada em laboratório, contando com o suporte da professora e da outra aluna de mestrado. No segundo dia de avaliações, já abrangendo a parte 2 do conteúdo, o G1 participou de outra atividade desplugada, diferente da primeira, e o G2, que havia participado da primeira atividade desplugada, respondeu a uma prova objetiva. As duas atividades desplugadas e as duas provas eram diferentes e sobre conteúdos distintos (Figura 1).

Optou-se por dividir a turma em dois grupos para que fosse possível auxiliar efetivamente os alunos por ocasião das atividades desplugadas, assim como observar a interação deles no desenvolvimento das atividades. As provas objetivas foram realizadas individualmente, enquanto as atividades desplugadas foram desenvolvidas em equipes de 4 alunos cada, pois o objetivo era identificar os benefícios do uso da CD e do tra- 


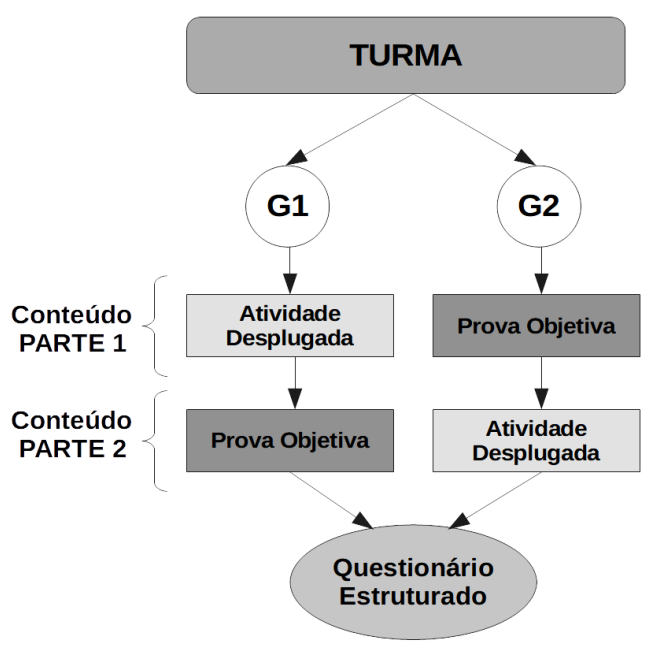

Figure 1. Metodologia das atividades

balho em grupo em relação às práticas tradicionais de avaliação individuais. A respeito da avaliação de aprendizagem (abordagens, teorias, métodos) é importante afirmar que a forma de avaliação tem impacto nos resultados e análise: uma avaliação por prova objetiva individual é muito diferente de uma avaliação por meio de uma atividade desplugada em grupo, na qual nesta última, há a interação entre os membros da equipe, comunhão de esforços e compartilhamento de conhecimento. O conteúdo programático aferido nas avaliações contemplaram banco de dados relacionais e foram dividos em: parte 1 - operações DML (Data Manipulation Language) e DQL (Data Query Language) da linguagem SQL (Structure Query Language), escalonamento de transações, seriabilidade de escalonamentos e problemas de controle de concorrência em transações; parte 2 - escalonamento de transações, técnicas de bloqueio dos itens de dados, starvation, deadlocks e protocolos para evitá-los.

\subsection{Atividades Desplugadas}

A atividade desplugada referente à parte 1 do conteúdo foi dividida em três etapas: definição das transações (comandos em DML e DQL), escalonamento 1 com problema e ajuste deste escalonamento para que o problema fosse solucionado, gerando um escalonamento 2. Ou seja, os alunos precisavam definir 2 transações, baseadas no modelo lógico de um banco de dados fornecido previamente, obter mais 2 transações de outra equipe e intercalá-las para gerar, inicialmente, um escalonamento intercalado, recuperável e que apresentasse o problema de controle de concorrência chamado agregação incorreta. Após isso, os alunos deveriam transformá-lo em um escalonamento em cascata e/ou estrito, indicando se os 2 escalonamentos gerados eram seriáveis em conflito.

Nesse contexto, primeiramente as equipes atuaram como se fossem uma aplicação interagindo com um banco de dados, definindo um conjunto de operações DML e DQL. A criação das transações deveriam atender a certos requisitos, sendo estes: cada equipe teve que criar pelo menos duas transações, sendo cada uma composta por no mínimo duas e máximo cinco operações, e ao menos uma delas deveria ser um comando DML (insert ou update) e um DQL (select). Além disso, uma das transações deveria ter uma função de agregação (sum, count, max, min e $a v g$ ). Ao término da criação, as transações 
foram compartilhadas de modo que cada equipe recebeu duas transações de outra equipe, totalizando 4 transações para serem traduzidas na etapa seguinte.

Posteriormente, na etapa 2, as equipes trabalharam como um Sistema Gerenciador de Banco de Dados (SGBD) e precisaram traduzir as transações para comandos de leitura e escrita, além de escalonar esses comandos de maneira intercalada pois é dessa forma que o SGBD relacional realiza o processamento das transações. O primeiro escalonamento apresentado deveria ser completo (finalizar as transações com um commit ou abort), conter obrigatoriamente o problema de controle de concorrência chamado agregação incorreta e ser do tipo recuperável, ou seja, havia a necessidade de uma das transações conter um função de agregação para que o escalonamento apresentasse o problema pedido. Ser recuperável diz repeito à uma característica do escalonamento em que uma transação $t$ só pode ser confirmada (commited) se todas as transações $t^{\prime}$, que tenham gravado um item de dados que $t$ leia, também tenham sido confirmadas. Na etapa 3, era necessário transformar o escalonamento 1 (recuperável) em um escalonamento 2, sem rollback em cascata. Isso quer dizer que no novo escalonamento, a reversão de uma transação não deveria acarretar o retrocesso de outras transações. Além disso, o escalonamento 2 poderia ser estrito também, ou seja, não apresentar transações que realizassem leitura ou gravação de itens de dados, até que a última transação que gravou esse mesmo item tenha sido confirmada. Ao final, era necessário indicar através de um gráfico direcionado, se os escalonamentos 1 e 2 eram seriáveis em conflito.

Os alunos construíram a resolução usando papel A3, post-its (adesivos) coloridos, caneta hidrocor, cola e tesoura. Eles precisavam representar as operações de leitura/escrita e funções de agregração nos post-its coloridos, mantendo a padronização de usar sempre a mesma cor de adesivo para as operações. Após escrever as operações nos adesivos, eles organizaram os 2 escalonamentos nas folhas A3, indicando ao final se eram serializáveis ou não. A notação utilizada ficou à cargo de cada equipe e as dúvidas sanadas no decorrer da atividade, restringiram-se à melhor forma de representar a solução para que a professora tivesse o entendimento do que ali estava representado. Os alunos não podiam interagir com outras equipes, apenas com seus próprios integrantes. A Figura 2 mostra um escalonamento na qual a equipe convencionou utilizar os post-its amarelos para leitura e rosas para a escrita. As colunas no topo da página, indicadas por $T_{1}$ a $T_{4}$, são as quatro transações. A linha do tempo para a execução das operações inicia com a escrita (write) do item "Negocio" pela transação $T_{2}\left(w_{2}(\right.$ Negocio $)$ ) e segue na sequência intercalada das operações (de cima para baixo). As operações $r_{x}$ indicam leitura (read) e o $x$ é o número identificador da transação. O escalonamento é completo, pois todas as transações são finalizadas com o commit. A equipe indicou ao final que o escalonamento não é seriável pois o gráfico apresenta um ciclo, porém o grafo apresentado não ilustra essa característica.

A atividade desplugada realizada pelo G2, abordou a parte 2 do conteúdo e dividiu-se em três etapas também: definição das transações/bloqueio dos itens, escalonamento 1 (intercalado e completo) e escalonamento 2 para evitar impasses (deadlocks). A dinâmica foi bastante parecida com a atividade anterior. Na etapa 1, as equipes simularam a interação entre uma aplicação e o SGBD, pois precisaram definir 2 transações usando comandos de leitura e escrita, baseadas no mesmo modelo lógico da atividade desplugada do G1. Obtiveram 2 transações de outra equipe, inseriram os bloqueios dos itens 


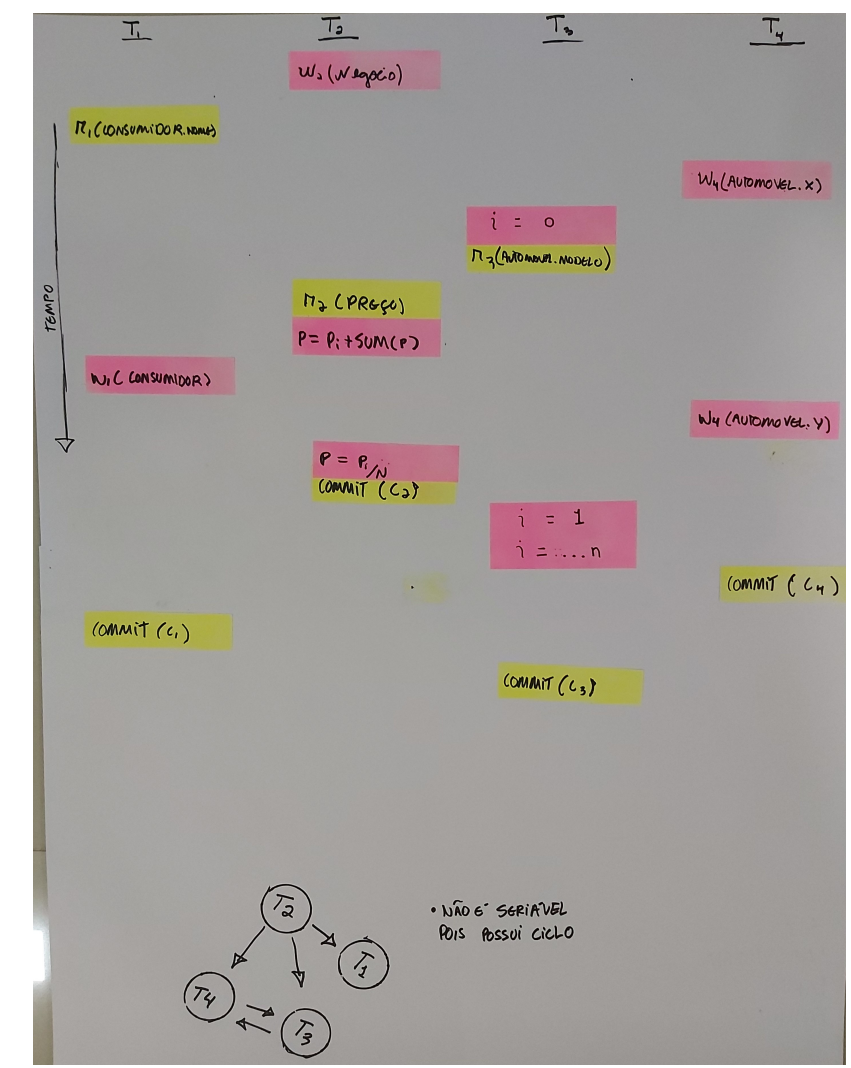

Figure 2. Resolução do Escalonamento 1 da primeira atividade desplugada.

através do protocolo em duas fases (two phase locking) rigoroso. Nesta atividade, não houve a necessidade de traduzir os comandos em SQL para leituras e escritas, mas em compensação foi preciso inserir marcadores indicando o bloqueio (lock) e desbloqueio (unlock) de itens, antes deles serem acessados pelas operações de leitura/escrita.

$\mathrm{Na}$ etapa 2, era necessário intercalar as operações das 4 transações e gerar um escalonamento 1, não serial e completo. A equipe funcionou como um SGBD, gerenciando as transações e tentando garantir o controle de concorrência, porém era preciso analisar o escalonamento 1 para verificar se havia a possibilidade de gerar impasses ou espera indefinida (starvation). Os impasses são situações que podem ocorrer por conta da disputa por acessos a itens de dados e, neste caso, uma transação $t$ já possui bloqueio para acessar um item de dados $a$ e solicita o bloqueio de um outro item $b$. Entretanto, esse item $b$ já está bloqueado por uma outra transação $t^{\prime}$ que requer o item $a$, já bloqueado por $t$. Em contrapartida, a espera indefinida ocorre quando uma transação espera por bloquear um item, sendo que essa espera pode ser indefinida pois não há garantia de que ela conseguirá o bloqueio do item que precisa para seguir sua execução. Caso o escalonamento 1 apresentasse impasse, a equipe deveria ajustá-lo, gerando um escalonamento 2 (etapa 3). Para isso, era necessário usar o protocolo multiversão com ordenação por timestamps ou o bloqueio em duas fases usando certificação. Nesta atividade desplugada, referente ao conteúdo - parte 2, as equipes utilizaram o mesmo material que o G1 utilizou e a dinâmica foi a mesma: precisaram representar as operações das transações através de post-its, utilizando as mesmas cores para as operações iguais, e ao final apresentaram 2 escalonamentos. A forma de representação também foi livre, as dúvidas sanadas eram 
IX Congresso Brasileiro de Informática na Educação (CBIE 2020)

Anais do XXVI Workshop de Informática na Escola (WIE 2020)

apenas sobre a forma de representação das transações e era permitida a interação apenas entre os membros da mesma equipe.

\section{Avaliação e Resultados}

A avaliação das atividades e desempenho dos alunos aconteceu da seguinte forma: habilidade dos alunos ao aplicarem os conteúdos vistos na disciplina na resolução das atividades desplugadas, prova objetiva e preenchimento de um questionário estruturado, para colher informações sobre o grau de satisfação dos alunos e dificuldades em participar de um instrumento de avaliação baseado em Metodologias Ativas. As provas objetivas (Partes 1 e 2) foram realizadas individualmente e continham três questões cada, totalizando 5,0 pontos. Da mesma forma, as atividades desplugadas foram divididas em três etapas, conforme descrito na seção anterior, valiam 5,0 pontos e, somados às provas objetivas, compreendiam os 10,0 pontos referentes ao valor total da segunda avaliação. Os resultados referentes às avaliações sobre o conteúdo da parte 1, mostraram que os alunos participantes da atividade desplugada conseguiram expressar melhor o conceito abstrato de transações, tiveram mais clareza em aplicar os algoritmos de escalonamento e verificar a seriabiliade deles. Isso refletiu na nota desses alunos, pois eles obtiveram rendimento $29 \%$ melhor do que aqueles que realizaram prova objetiva. Na avaliação referente à parte 2 do conteúdo, observou-se a mesma porcentagem de melhora no rendimento dos alunos que participaram da atividade desplugada, ou seja, eles conseguiram aplicar de maneira mais efetiva os algoritmos de escalonamento, simular o bloqueio de itens pelas transações e verificar se ocorreram impasses ou espera indefinida.

Durante o desenvolvimento das atividades, foi observada uma forte cooperação entre os integrantes da equipe, somando esforços para elaborar a solução das questões, e alguns alunos destacaram-se como líderes, definindo as atividades de cada membro e organizando a dinâmica de trabalho. Essa cooperação do trabalho em equipe é uma das hipóteses para a melhora no redimento dos alunos. A outra hipótese é o fato das atividades envolverem artefatos físicos, facilitando a representação real das transações, concretização das estratégias para a resolução das atividades, além de um maior tempo para realizá-las. Os alunos responderam a um questionário online, baseado na escala Likert. Essa escala foi construída por Rensis Likert e, em cada questão, avalia-se o grau de concordância ou discordância sobre algo, considerando geralmente cinco gradações: concordo muito,concordo, neutro/indiferente, discordo, discordo muito [Aguiar et al. 2011]. O questionário serviu para avaliar, sob a perspectiva dos discentes, a metodologia adotada, o desenvolvimento das atividades desplugadas em si, além de identificar o nível de satisfação e aceitabilidade da turma em realizar uma atividade avaliativa não tradicional.

Quanto ao nível de conhecimento dos alunos a respeito de $\mathrm{CD}, 69.2 \%$ dos alunos que responderam ao questionário, mostraram não obter nenhum conhecimento prévio, ao mesmo tempo que $85 \%$ nunca havia participado de atividades desplugadas. Apesar da maioria dos estudantes não terem tido nenhum tipo de conhecimento ou vivência prévia com a CD, mais de $60 \%$ da turma demonstrou satisfação ao realizar as atividades propostas e em participar de outras atividades futuras com as mesma metodologia. Quanto à contribuição da CD para melhor expressar os conteúdos compreendidos, quase $80 \%$ dos alunos afirmaram que a atividade contribuiu positivamente, o que mostra o quão atraente e benéfica é esse tipo de metodologia para alunos do ensino superior. A respeito do nível de dificuldade da atividade, $42.3 \%$ dos alunos informaram ser uma atividade de média difi- 
culdade, enquanto que 53.9\% afirmou ser uma atividade difícil. É importante ressaltar que os conteúdos cobrados são considerados bastante complexos, por exigir um alto nível de abstração dos alunos. $\mathrm{O}$ apoio docente e o trabalho em grupo mostraram ser muito importantes para as atividades, segundo mais de $80 \%$ da turma. Setenta e sete porcento $(77 \%)$ dos alunos afirmaram que o material disponibilizado para a atividade era satisfatório, já o tempo disponível não foi suficiente, segundo $38.5 \%$ dos alunos. Os dados obtidos através do questionário mostram que a maioria dos alunos desconheciam as Metodologias Ativas e Computação Desplugada, porém gostaram de participar das atividades e participariam novamente em outra oportunidade.

\section{Conclusão}

Embora o uso de atividades desplugadas seja mais comum na educação básica, elas podem ser adaptadas para serem aplicadas no ensino superior. Esta pesquisa objetivou apresentar um relato de experiência na disciplina de BD II em uma turma de Ciência da Computação. Os resultados mostraram que as atividades desplugadas contribuíram para que os alunos expressasem melhor a compreensão sobre os conceitos abstratos vistos na disciplina e aplicassem na prática, através de objetos físicos, os algoritmos e estratégias utilizadas pelos SGBDs para gerenciar as transações e realizar o controle de concorrência para acessar itens de dados. As aulas teóricas prévias, o trabalho em grupo e a mediação da professora foram importantes para a realização da atividade desplugada, refletindo na nota geral da segunda avaliação, ultrapassando a média mínima para aprovação (5.0 pontos). Em comparação com a metodologia de avaliação tradicional (prova escrita), a atividade desplugada resultou em melhor redimento dos alunos, visto que a média das notas dessas atividades mostrou-se $40 \%$ maior em detrimento à prova objetiva, porém os alunos relataram que seria mais adequado ter mais tempo para realizar a atividade devido ela ser um instrumento avaliativo. Vale ressaltar que a avaliação da aprendizagem dos estudantes, precisa ir além do simples ato de dar uma nota, mas sim levar em consideração o processo de construção do conhecimento.

Perante o exposto, as atividades desplugadas no ensino superior podem ter uma ótima receptividade por parte dos alunos, se aplicadas de maneira adaptada à realidade e ao contexto das disciplinas. Ressalta-se que esses resultados são consequência da junção da $\mathrm{CD}$ e do trabalho em equipe, portanto como trabalhos futuros indica-se a comparação entre atividades desplugadas de avaliação em grupo e de atividades desplugadas de avaliação individuais. Além disso, espera-se a elaboração de mais atividades desse tipo para que as mesmas sejam utilizadas como metodologia de ensino e não apenas como instrumento avaliativo, auxiliando os alunos em uma melhor compreensão e fixação dos assuntos abordados.

\section{References}

Aguiar, B., Correia, W., and Campos, F. (2011). Uso da escala likert na análise de jogos. In SBC - Proceedings of X SBGames, pages 01-05. SBC.

Alejandro Melgaço, J. D. (2019). Desenvolvimento e aplicação de práticas desplugadas para o ensino de redes de computadores. In Anais do XXV Workshop de Informática na Escola, pages 481-490. SBC.

Assis, P. (2015). Educação e tecnologias: o novo ritmo da informação. Bolema Boletim de Educação Matemática, pages 428-434. 
IX Congresso Brasileiro de Informática na Educação (CBIE 2020)

Anais do XXVI Workshop de Informática na Escola (WIE 2020)

Bell, T., Witten, L., and Fellows, M. (2011). Computer Science Unplugged: Ensinando Ciência da Computação sem o uso do computador. Computer Science Unplugged ORG.

Berardi, R., Kozievitch, N., Bim, S., and Auceli, P. (2019). Oficina de banco de dados com aprendizado cinestésico para meninas do ensino médio. Anais do XXV Workshop de Informática na Escola, pages 345-354.

Berto, L., Zaina, L., and Sakata, T. (2019). Metodologia para ensino do pensamento computacional para crianças baseada na alternância de atividades plugadas e desplugadas. Revista Brasileira de Informática na Educação, 27(02):01-10.

Chris, J. and Shao, B. (2011). The net generation and digital natives: Implications for higher education. Higher Education Academy York, pages 1-57.

Curzon, P., McOwan, P., Plant, N., and Meagher, L. (2014). Introducing teachers to computational thinking using unplugged storytelling. In Proceedings of the 9th workshop in primary and secondary computing education, pages 89-92.

Gonçalves, D. A. S., da Silva, G. M., da Luz, R. S., and Silva, C. E. P. (2013). Relato de experiência de alunos do curso de licenciatura em computação do ifmg-campus ouro branco na utilização de objetos de aprendizagem desplugados e do scratch como instrumentos no ensino de programação. In Anais dos Workshops do II Congresso Brasileiro de Informática na Educação, pages 335-344. SBC.

Hartwig, A., Silveira, M., Fronza, L., Silveira, H., Mattos, M., and Kohler, L. (2019). Metodologias ativas para o ensino na graduação na area de computação. In Anais do XXVIII Workshop de Informática na Escola, pages 1139-1143. SBC.

Lázaro, A. C., Sato, M. A. V., and Tezani, T. C. R. (2018). Metodologias ativas no ensino superior: O papel do docente no ensino presencial. Anais CIET:EnPED:2018 Educação e Tecnologias: Docência e mediação pedagógica, pages 1-12.

Martinhago, A., Smarzaro, R., Lima, I., and Guimarães, L. (2014). Computação desplugada no ensino de bancos de dados na educação superior. In Anais do XXII Workshop sobre Educação em Computação, pages 21-29, Porto Alegre, RS, Brasil. SBC.

Moreira, J. and Monteiro, W. (2018). O uso da computação desplugada em um contexto de gamificação para o ensino de estrutura de dados. RENOTE, 16:546-555.

Prodanov, C. C. and Freitas, E. C. (2013). Metodologia do trabalho científico: métodos e técnicas da pesquisa e do trabalho acadêmico-2 ${ }^{a}$ Edição. Editora Feevale.

Reis, R., Lyra, K., Reis, C. D. G., and Isotani, S. (2018). Relato de experiência sobre o uso da computação desplugada associada a uma teoria de aprendizagem colaborativa. pages $166-175$.

Silva, D., Silva, R., and Coutinho, C. (2018). Ensinando redes de computadores utilizando metodologia ativa e a computação desplugada. In Anais do XVIII Escola Regional de Computação Bahia, Alagoas e Sergipe, pages 428-437, Porto Alegre, RS, Brasil. SBC.

Souza, F., Leite, R., Brito, C. M., Villela, M., and Santos, C. Q. (2019). O desenvolvimeno do pensamento computacional além do ensino em ciências exatas: uma revisão da literatura. In Anais XXX Simpósio Brasileiro de Informática na Educação - SBIE, pages 528-536. SBC. 